\title{
Enhancing cancer clonality analysis with integrative genomics
}

\author{
Erich A Peterson, Michael A Bauer, Shweta S Chavan, Cody Ashby, Niels Weinhold, Christoph J Heuck, \\ Gareth J Morgan, Donald J Johann*
}

From 12th Annual MCBIOS Conference

Little Rock, AR, USA. 13-14 March 2015

\begin{abstract}
Introduction: It is understood that cancer is a clonal disease initiated by a single cell, and that metastasis, which is the spread of cancer from the primary site, is also initiated by a single cell. The seemingly natural capability of cancer to adapt dynamically in a Darwinian manner is a primary reason for therapeutic failures. Survival advantages may be induced by cancer therapies and also occur as a result of inherent cell and microenvironmental factors. The selected "more fit" clones outmatch their competition and then become dominant in the tumor via propagation of progeny. This clonal expansion leads to relapse, therapeutic resistance and eventually death. The goal of this study is to develop and demonstrate a more detailed clonality approach by utilizing integrative genomics.
\end{abstract}

Methods: Patient tumor samples were profiled by Whole Exome Sequencing (WES) and RNA-seq on an Illumina HiSeq 2500 and methylation profiling was performed on the Illumina Infinium 450K array. STAR and the Haplotype Caller were used for RNA-seq processing. Custom approaches were used for the integration of the multi-omic datasets.

Results: Reported are major enhancements to CloneViz, which now provides capabilities enabling a formal tumor multi-dimensional clonality analysis by integrating: i) DNA mutations, ii) RNA expressed mutations, and iii) DNA methylation data. RNA and DNA methylation integration were not previously possible, by CloneViz (previous version) or any other clonality method to date. This new approach, named iCloneViz (integrated CloneViz) employs visualization and quantitative methods, revealing an integrative genomic mutational dissection and traceability (DNA, RNA, epigenetics) thru the different layers of molecular structures.

Conclusion: The iCloneViz approach can be used for analysis of clonal evolution and mutational dynamics of multi-omic data sets. Revealing tumor clonal complexity in an integrative and quantitative manner facilitates improved mutational characterization, understanding, and therapeutic assignments.

\section{Background}

It is recognised that cancer is a clonal disease instigated by a single cell and that metastasis is also commenced thru a single cell [1-3]. Tumors are composed of a variety of clones or subpopulations of cancer cells that may differ, for instance in their expression of cell surface markers, sensitivity to therapeutic agents, karyotype, proliferation rate. A cancer clone or subclone is a cell or group of cells that have formed from an original cell as a result of a new

\footnotetext{
* Correspondence: don.johann@gmail.com
}

Myeloma Institute, University of Arkansas for Medical Sciences, Little Rock, AR, USA

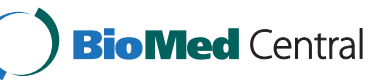

mutation [4]. Many cancers including multiple myeloma $(\mathrm{MM})$ are difficult to treat due to their dynamic adaptability resulting from clonal evolution [5].

Evolution is an important scientific concept because it works. It provides a framework to explain changes in biological systems. Cancer is the result of an evolutionary process, but it is destructive, since it involves the loss of mechanisms that are implemented to protect against uncontrolled and undifferentiated growth. Ultimately, natural selection has a harsh reality that worried Darwin, namely, all that seems to matter is reproductive success [6]. 
MM is a cancer of the bone marrow characterized by a malignant transformation and proliferation of plasma cells [7]. Definitive therapies include combination chemotherapy, autologous transplant regimens [8], and two new classes of agents called immunomodulatory drugs (IMiDs) and proteasome inhibitors [9-11]. A significant improvement in patient survival has occurred over the last $\sim 15$ years [12]. However, there is still significant variation in outcome and an explanation is tumor heterogeneity with associated complex genomic landscapes $[5,13,14]$.

There exist an array of computational methods/tools that allow one to characterize various aspects of the clonal architecture of a tumor(s). Each method employs different computational and visualization techniques, and are very briefly described. SciClone [15] allows for the characterization of the clonal structure of a tumor using multiple samples, in an attempt to shed light on "cryptic subclones", which can appear when only one sample is analyzed. Several visualizations are available within SciClone, and clustering on the variant allele frequencies of somatic mutations (using a variational Bayesian mixture model on copy number neutral regions) attempts to infer the number and composition of subclones. PyClone [16] makes use of hierarchical Bayesian clustering models to estimate the number and cellular prevalence of subclones from the variant allele frequencies of somatic mutations. The method also takes into account copy number variation and possible normal cell contamination in the model. TrAp [17], in addition to inferring the subclones and their abundances within a single "aggregate sample" (via "aberrant frequencies" aka variant allele frequencies), constructs a phylogenetic tree-describing the evolution of clones within the tumor. The problem is modeled, and solutions based mathematically, on the deconvolution of a single aggregate signal.

Clomial [18] is another method used to infer subclonal structure using a binomial expectation maximization based approach (via somatic variant allele frequencies), and was specifically designed to deal with multiple samples from a single tumor sample. Rec-BTP [19] casts the problem of uncovering the clonal structure of a single sample, using the variant allele frequencies of somatic mutations, as a combinatorial one. A recursive algorithm using a binary tree partition is developed which approximates the originally formulated NP-complete problem. THetA [20] uses copy number aberrations to discover most likely clonal subpopulations. In particular, the problem of subclonal characterization is solved using a maximum likelihood mixture decomposition method, in order to find the genotypes "whose mixture best explains the observed sequencing data." PhyloWGS [21] is unique, in that it makes use of both somatic mutational and copy number variation data in its subclonal analysis. It additionally infers a phylogenetic tree (using non-parametric mathematical approaches) explaining the evolutionary history of a tumor's clonal composition, as well as, each clone's relative abundance. The method is also able to take multiple sample inputs to aid subclonal reconstruction.

All of the aforementioned referenced methods only make use of a single modality in their characterizations of clonal architecture, namely, DNA-based mutational data, culled from whole genome or whole exome sequencing (WES) experiments. Some methods also attempt to account for the effect of copy number variation on clonal architecture. This is contrasted to the multiple modality datasets employed in the characterization of clonal structure used within iCloneViz. iCloneViz is the only known computational method for inferring clonal architecture, which integrates multiple modality datasets to derive deeper biological meaning. For example, RNA variant calling is performed (a relatively new method of variant calling) to detect whether or not a mutation found within the DNA (via a WES or WGS experiment) is detectable within a RNA transcript. Further, if a mutation is found to be present at the RNA level, the expression values associated with the transcript(s) containing the mutation can be quantified and visualized. Finally, DNA methylation data is integrated into the analysis, which could lead to hypotheses regarding methylation suppressing the expression of a tumor suppressor gene (TSG). iCloneViz tracks TSGs both by mutations and epigenetic/methylation events.

In the presented analysis, integrated clonal dynamics from a single patient diagnosed with $M M$ is explored. Tumor material is investigated at initial diagnosis (i.e., Presentation) and then later when the cancer recurred (i.e., Relapse). A novel bioinformatic approach named CloneViz [22] has been enhanced to allow for the rapid integration, quantitation, visualization, and investigation of the mutational dynamics from i) WES, ii) RNA-seq, and iii) DNA methylation.

The methodology is independent of any specific cancer type and MM is used as a demonstrative example due to its intrinsic heterogeneity. The novelty of the approach concerns the rapid integration and dissection of large and complex multi-omic datasets. Discovered in this study is first, the existence of an amplified and mutated $M Y C$ oncogene in both Presentation and Relapse. Second, the occurrence of hotspot mutations in MTOR with rationale to treat, and in KRAS, with evidence not to treat. Third, is progressive evidence of tumor suppressor gene silencing. Overall, the molecular profiling and advanced bioinformatics (iCloneViz) provide for a more precise understanding of tumorigenesis with potential for an improved outcome via precision medicine-based approaches.

\section{Methods}

\section{Sample and library preparation}

All samples were obtained from a single patient with MM at the Myeloma Institute, University of Arkansas for 
Medical Sciences (UAMS), Little Rock, AR. Sample collection protocols were approved by the UAMS Institutional Review Board (IRB). Collected samples include bone marrow aspirate with biopsy and peripheral blood. Tumor plasma cells were obtained from the bone marrow aspirate after enrichment by anti-CD 138 immunomagnetic bead selection at a central laboratory, in a manner previously described [23]. Selected tumor material had a purity greater than $96 \%$ for the presentation sample and $99 \%$ for relapse. CD-138 is a marker for malignant plasma cells in a patient with clinical MM. The patient's first (Presentation) and second (Relapse) tumor samples were obtained in 2010 and 2013 respectively. Normal (germline) material was obtained from the buffy coat of peripheral blood in 2010, after density gradient centrifugation. To ensure the absence of plasma cells buffy coat material was also examined by flow cytometry.

Tumor and normal whole exome libraries were constructed from $50 \mathrm{ng}$ of DNA material after shearing, end repair, phosphorylation, and ligation to bar coded sequencing adapters. DNA material was further fragmented using the S220 focused-ultrasonicator (Covaris), for a target base pair (bp) length of 300. DNA was size selected for lengths between $\sim 250-330 \mathrm{bp}$. DNA regions were captured using the Agilent SureSelect ${ }^{\mathrm{QXT}}$ Human All Exon v5 Plus hybrid capture kit. Samples were then multiplexed and subjected to sequencing (101 bp paired-end reads) on an Illumina HiSeq 2500. Whole exome libraries were sequenced to an average depth of 100x.

RNA-seq tumor libraries were constructed using $200 \mathrm{ng}$ of total RNA material, using the Illumina TruSeq mRNA v2 kit according to the manufacturer's instructions. Poly-A selection for mRNA was first performed using streptavidin-coated magnetic beads, which was followed by thermal mRNA fragmentation. mRNA was then subjected to cDNA synthesis using reverse transcriptase. Resulting cDNA was converted to double stranded cDNA, followed by end repair, and then ligated to paired-end adapters. Size selection was performed using AMPure XP beads (Beckman Coulter), for sequences $\sim 300-350 \mathrm{bp}$ in length. The library was further enriched using 15 cycles of PCR and purified again with AMPure XP beads. The concentration of material run was $8 \mathrm{pM}$. The libraries were then multiplexed and subjected to sequencing (101 bp paired-end reads) on an Illumina HiSeq 2500. RNA-seq libraries were sequenced to a total of $100 \mathrm{M}$ reads.

Genome-wide DNA methylation was assessed in bisulfite-converted genomic DNA using the Illumina Infinium HumanMethylation450 (HM450K) BeadChip array, which contains 485,577 probes covering $99 \%$ of RefSeq genes, 96\% of CpG islands (CGI) and coverage across promoters, 5' and 3'-UTRs, first exons and gene bodies. Genomic DNA (500 ng) was bisulfite treated and purified using the EZ DNA Methylation-Gold kit
(Zymo Research, Irvine, CA) according to the manufacturer's protocol. The resultant bisulfite-converted DNA was processed, hybridized to Illumina HumanMethylation450 BeadChips, fluorescently stained and scanned according to the Infinium HD Assay Methylation Protocol User's Guide provided by Illumina. Processed BeadChips were scanned on an Illumina iScan and methylation values were determined for all probes using the GenomeStudio Methylation module (Illumina).

\section{Whole exome sequencing processing}

FASTQ file generation and demultiplexing from BCL files was performed using CASAVA v1.8.2 [24]. Quality control and assessment was performed on FASTQ files using FastQC v0.11.2 [25]. Unidentified bases at read ends (i.e., those recorded as ' $N$ ') were removed using a custom utility-creating final read lengths between 93 to 101 bps. Each sample's FASTQ paired-end files were aligned to the Ensembl reference genome (build GRCh37.75) using a hybrid approach that employed BWA v0.7.12 [26] and then STAMPY v1.0.22 [27]. Quality control and assessment of the aligned sequence alignment/map (binary alignment/map) SAM/BAM files were assessed with QualiMap v2.0.2 [28]. SAM/BAM post-processing steps were performed to mark duplicates, add read group information, sort, and reorder aligned reads (Picard Tools v1.119 [29]). GATK v3.3-0 [30] was used to perform local realignment and base quality recalibration. Copy number data was computational inferred using ExomeCNV v1.4 [31]. Single nucleotide variants (SNVs) and small insertions and deletions (InDels) were called using Strelka v1.0.14 [32] (tumor and normal pairs), resulting in variant call format (VCF) files. SnpEff v4.0e [33] was used to annotate each variant with its predicted functional effects.

\section{RNA sequencing processing \\ Transcriptome reconstruction}

RNA-seq samples were first demultiplexed and FASTQ files were created from BCL files using CASAVA v1.8.2. Quality control and assessment was performed on FASTQ files using FastQC v0.11.2. Trimmomatic v0.32 [34] (utilizing a sliding window approach) was used to trim low quality reads and remove possible adapter sequences. Alignment of reads and transcriptome reconstruction was performed using the Tuxedo suite of tools. TopHat v2.0.12 [35] was used to align each sample's paired-end reads to the Ensembl reference genome build GRCh37.75. Quality control and assessment of resulting BAM files was performed using SAMtools v0.1.8 [36]. BAM files were then used to reconstruct the transcriptome and quantify each isoform's fragments per kilobase of transcript per million mapped reads (FPKM) using Cufflinks v2.2.1 [37], and Cufflinks was run in a mode 
which allows for the discovery of novel isoforms. HTSeq v0.6.1 [38] was used to quantify raw (non-normalized) gene-based read counts.

\section{RNA-seq based variant calling}

After demultiplexing and creating FASTQ files using the previous subsection's description, RNA variants were called using the Broad Institute's GATK Best Practices for RNA-seq variant calling [39]. These steps include the following: STAR v2.3.0e [40] was used to align reads to the Ensembl reference genome (build GRCh37.75), using the recommended "2-pass" approach. Duplicates were marked and the aligned reads sorted with Picard Tools. Next, the tool SplitNCigarReads (GATK component) was used to split reads into exon segments, clip reads which overhang intronic regions, and assign a default MAPQ score of 60 to all reads. Variants were called using the HaplotypeCaller tool (GATK component).

\section{Variant quantification and classification}

The variant allele frequency (VAF) was determined by dividing the total reads for the variant (TRV) by the sum of the total reads for the variant (TRV) plus total reads for the reference (TRR). Copy number data was derived using ExomeCNV v1.4. The selection and retention of variants were based on the following filtering parameters: i) $\mathrm{VAF} \geq 4 \%$, and ii) $20 \leq \mathrm{DP} \leq 1000$. A manual evaluation of the read alignments using the Integrative Genomics Viewer (IGV) v2.3.32 was also performed [41]. At times a second selection of variants utilized an intersection against a key gene (KG) list. The KG group was constructed from the following public sources: i) known drivers and cancer predisposition genes cited in Vogelstein, et. al. [42], ii) Foundation One Heme ${ }^{\mathrm{TM}}$ Genes (http://foundationone.com/genelist2.php) and, iii) the MD Anderson listing of human DNA repair genes [43].

\section{Quantifying clonal diversity}

Diversity measures from ecology were adapted to quantify clonal diversity in MM serial samples [44]. Each sample is not a single organism/species, but rather consists of thousands of cells from a purified bone marrow aspirate. The abundance of a molecular species (variant/mutation) is the product of $V A F * D P * C N$. The number of clones in a neoplasm is a simple measure of diversity. Diversity measures typically incorporate both the number and abundance of clones [44]. The Shannon diversity index (SDI) [45] is

$$
S D I=-\sum_{i}^{N} p(i) \ln (p(i))
$$

where $p(i)$ is the frequency of clone $i$ in the neoplasm. The SDI computes a single quantitative value based on the number of different mutations in the cancer sample and how evenly distributed each mutation is among the entire group. The SDI value will increase when the number of distinct mutations increases and also when the evenness among the mutations increases [44]. There are other diversity measures (e.g., Simpson, Berger-Parker) but, the Shannon diversity index is preferable because it is not dominated by the most frequent clone, and it has been utilized in previous studies of cancer [46,47].

\section{Software engineering and integrative analysis}

This section and its constituent sub-sections documents and describes all software components, data structures, and algorithms used to construct the extended and integrated CloneViz (iCloneViz), especially the data calculations, visualizations and the rationale for their use.

\section{General technology and frameworks}

The C\# programming language, targeting the .NET Framework v4.5 [48] (utilizing the integrated development environment Visual Studio 2013 [49]), was used to construct iCloneViz. This programming platform was used to facilitate the rapid development of an interactive Windows-based application. To minimize the amount of custom data access layer code needed to perform the dataintensive functions of iCloneViz, the object-relational mapping software Entity Framework v6.1.1 [50] (using the database-first model) was utilized along with the molecular profiling modality database (MPMDB), which is further described later in this study. The built-in .NET Framework charting package (i.e., namespace System. Windows.Forms.DataVisualization.Charting), was used to provide visualization primitives. This package was used in favor of others, because of its relative ease-of-use and built-in functions, in particular, its ability to perform smart label positioning. The relational database management system SQL Server 2012 Developer Edition [51] was used to store all patient data and meta-data. The database system was chosen for its robustness, ability to scale, and its low cost for academic and research use. (The database system is described in further detail in the "Data storage, retrieval and annotation" section below).

\section{Mathematics and statistics}

To assist in kernel density estimation (KDE) and perform probability density calculations necessary to estimate the unknown distribution of the mutations found, the package Math.NET Numerics v3.2.3 [52] was used. The standard normal (Gaussian) kernel was used in the estimation. The equation used to estimate the probability density function induced by the mutations is as follows:

$$
\hat{f}_{h}(x)=\frac{1}{n h} \sum_{i=1}^{n} K\left(\frac{x_{i}-x}{h}\right)
$$

Where $x$ is the point at which density is to be estimated, $\left(x_{1}, x_{2}, \ldots, x_{n}\right)$ is the array of independent and identically distributed (i.i.d) sample (mutations) of some unknown distribution, $n$ is the array size, $h$ is the bandwidth, and $K$ is the standard normal kernel function. 
To account for the effect of copy number variation (CNV) on mutations found within regions containing copy number alterations, the following was done: i) copy number information was inferred using ExomeCNV for each loci of the tumor exome; ii) each mutation's loci was associated with those regions found in (i); iii) each mutation was weighted based on the copy number for the loci it lied within. The statistical software package R v3.1.3 [53], in particular, the KernSmooth (ks) package v1.9.4 [54] (hpi function) was used to calculate a data-derived univariate kernel density "plug-in" bandwidth [55]. The package R.NET v1.6 [56] was utilized to provide an application programming interface from .NET to $\mathrm{R}$ and the ks package.

\section{Data storage, retrieval and annotation}

At the Myeloma Institute the MPMDB is a pre-existing, research-driven molecular profiling repository. The design philosophy of the database was to provide a centralized information architecture for interfacing additional custom tools (e.g., iCloneViz) via various abstractions. The MPMDB provides many other features to facilitate the analysis of complex next generation sequencing (NGS) data, including extensive extract-transform-load (ETL) capabilities for data cleaning as well as data association and integration with various knowledge databases.

The integration of various modality datasets (aka tables or relations), which allow for the visualizations of $\mathrm{iCloneViz}$ are now described (N.B. not all attributes are defined for brevity). Standard database related theory and relational algebra notation are used [57]. A diagram titled, Multi-Omic Relational Integration (Additional File 1) illustrates the relations between the various relational algebra equations.

Let the schema of a relation be denoted as $A\left(a_{0}, a_{1}, \ldots\right.$, $\left.a_{n}\right)$, where $A$ is the relation's name and each $a_{i}(0 \leq i \leq n)$ is a name or identifier of an attribute within $A$ (the domain or data type for each attribute $a_{i}$ are excluded for brevity ).

Let:

i. $W$ be the resulting data from a whole exome sequencing experiment, that is, the SnpEff annotated results from the variant-calling application Strelka;

ii. $M$ be the data resulting from a methylation experiment;

iii. $C$ be the copy number data computationally inferred from a WES experiment using ExomeCNV; iv. $H$ be the raw count (unnormalized) data for each gene in an RNA-seq experiment using the htseqcount function from the HTSeq application;

v. $R V$ be the data resulting from RNA-seq variant calling using the Haloptype Caller (GATK);

vi. $R$ be RNA-seq transcript quantification data (FPKM) following transcriptome reconstruction by Cufflinks; vii. $K$ be a list of key genes representing cancer drivers and Variants of Uncertain Significance (VUS) the user is interested in labeling in each visualization; and viii. $P$ be patient and experimental meta-data.

The schema of each of the aforementioned relations to be integrated is defined as follows:

i. W (Chromosome, Position, DNADepth, DNAAllelicFreq, EnsemblGeneId, GeneSymbol, DNAChange, AminoAcidChange, EffectImpact, ExperimenId)

ii. M (TargetId, AvgBeta, Annotation, EnsemblGeneId, ExperimentId)

iii. C (Chromosome, Start, Stop, CopyNumber, ExperimentId)

iv. $H$ (EnsemblGeneId, Count, ExperimentId)

v. $R V$ (Chromosome, Position, EnsemblGeneId, RNADepth, RNAAllelicFreq, ExperimentId)

vi. $R$ (EnsemblTranscriptId, EnsemblGeneId, FPKM, ExperimentId)

vii. $K($ EnsemblGeneId $)$

viii. P (PatientId, FirstName, LastName, ExperimentDescription, ExperimentId, ExperimentDate, SampleType, SampleDescription, SampleCollectionDate)

Let $\bowtie_{A . a=B . b}$ denote the theta-join (in particular the equijoin) between relations $A$ and $B$ on attributes $a$ and $b$ respectively. Let $\bowtie_{L_{A . a B B . b}}$ denote the left-outer join between relations $A$ and $B$ on attributes $a$ and $b$ respectively. Let $\pi_{\left(a_{0}, \ldots, a_{n}\right)}$ denote the projection operator over attributes $\left(a_{0}, \ldots, a_{n}\right)$. Finally, let $\sigma_{E}(A)$ denote the selection operator, where $A$ is the relation to be selected from and $E$ is the conditional expression used for selecting tuples/rows from relation $A$. Given these definitions, and assuming each relation is selected for the experiment that is to be integrated, the final integration of the various multi-omic datasets can be defined as:

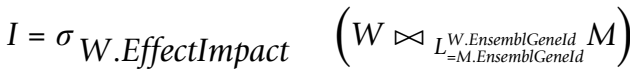

$$
\begin{aligned}
& { }^{\prime} \text { MODERATE }{ }^{\prime} \vee \\
& \text { W.EffectImpact } \\
& =^{\prime} H I G H^{\prime} \\
& J=\sigma_{R . F P K M>0}\left(\begin{array}{c}
R V \bowtie R V \text {.EnsemblGeneId } \\
=\text { R.EnsemblGeneId }
\end{array}\right) \\
& D=\sigma_{H . \text { Count }>0}\left(\begin{array}{c}
R V \bowtie_{R V \text {.EnsemblGeneId }} H \\
=H . \text { EnsemblGeneId }
\end{array}\right) \\
& L=I \bowtie L \quad \text { W.Chromosome }{ }^{J} \\
& =R V \text {.Chromosome } \wedge \\
& \text { W.Position } \\
& =R V \text {.Position }
\end{aligned}
$$




$$
\begin{gathered}
\begin{array}{c}
N=L \bowtie L \quad \text { W.Chromosome } \\
=R \text {.Chromosome }
\end{array} \\
\text { W.Position } \\
=\text { RV.Position } \\
\text { IntegratedRelation }=N \bowtie L \quad \text { W.Chromosome } C \\
=\text { C.Chromosome^ } \\
\text { W.Position } \geq \\
\quad \text { C.Start } \wedge \\
\text { W.Position } \\
\leq \text { C.Stop }
\end{gathered}
$$

Where IntegratedRelation denotes the final integrated relation. Given this final relation (and the key gene list $K$ to visually label), aggregations and selections can be performed to filter data and encode all visual aspects within iCloneViz. Some of those aggregations and selections are now defined for later reference:

$$
W_{1} \cap(\text { Chromosome,Position }) W_{2}
$$

Where $W_{i}$ denotes $\sigma_{W . E x p e r i m e n t I d=i}(W)$ and $\cap\left(a_{0}, \ldots, a_{n}\right)$ is the intersection operator based on common attributes $\left(a_{0}, \ldots, a_{n}\right)$.

$$
W_{1} \backslash(\text { Chromosome,Position }) W_{2}
$$

Where $\backslash\left(a_{0}, \ldots, a_{n}\right)$ denotes the set difference operator based on common attributes $\left(a_{0}, \ldots, a_{n}\right)$.

$$
\hat{W}=\operatorname{distinct}\left(\begin{array}{c}
\pi \text { Chromosome, } \\
\text { Position, } \\
\text { DNADepth, } \\
\text { CopyNumber, } \\
\text { DNAChange, } \\
\text { AminoAcidChange, } \\
\text { DNAAllelicFreq, } \\
\text { GeneSymbol, } \\
\text { Glyph }
\end{array}\right)
$$

Where Glyph $=$ [1: Only WES Data, 2: WES + RNA Data], is a computed attribute on IntegratedRelation indicating what type of data is available (not null) in each tuple of IntegratedRelation.

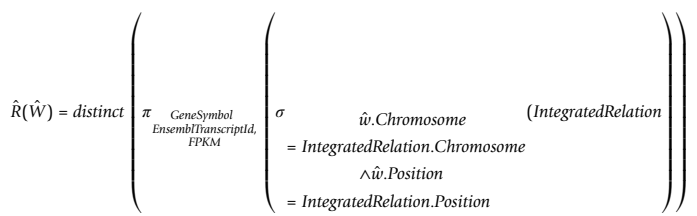

Where $\hat{w}$ is a tuple within relation $\hat{W}$ from Eq. (11).

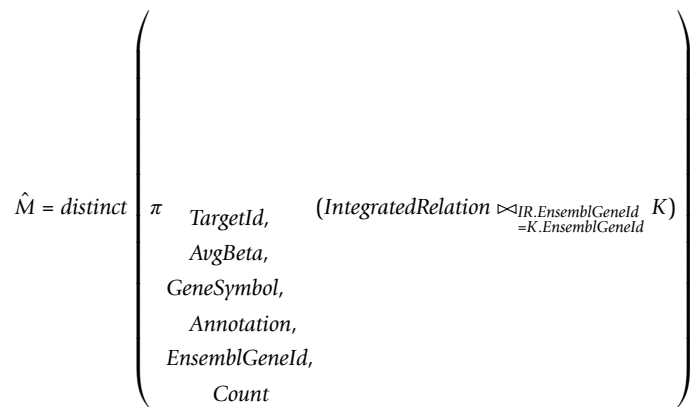

$$
\hat{R}=\operatorname{distinct}\left(\begin{array}{cc}
\pi & \\
& \\
& \text { GeneSymbol, } \\
& \text { RNADepth, } \\
\text { RNAAllelicFreq, } & \\
\text { FPKM, } \\
\text { EnsemblTranscriptId }
\end{array}\right)(14)
$$

Pseudocode describing the algorithms of iCloneViz can be found in the appendix.

\section{Results and discussion}

This study illustrates the integrated analysis of temporal-based clonal dynamics from a patient with MM. Examination of Presentation and Relapse samples from purified bone marrow aspirates are compared and contrasted using a bioinformatic approach named iCloneViz, which has been enhanced and redesigned to perform clonality analysis utilizing an integrative genomic (DNA, RNA, methylation) methodology, not previously possible. All graphs and visualizations in this study were generated by iCloneViz, unless indicated otherwise. It has been established that there is intraclonal heterogeneity at the level of single nucleotide variants (SNVs) in myeloma [58]. As a means to study the genetic heterogeneity and Darwinian nature that is germane to cancer, clonal analysis and integrative genomics has been advocated [59,60].

iCloneViz makes available a global view of all mutational events in a WES experiment. This is illustrated in Additional File 2. Subfigure A corresponds to the Presentation and $\mathbf{B}$ to the Relapse sample. The $\mathrm{x}$-axis 
contains an ordered list of chromosomes (1-22, X, Y). Each chromosomal region is sized by the number of base pairs it contains. The y-axis is organized by variant allele frequency (VAF). Each variant is a point on the plot and the color scale indicates the depth of coverage (depth). A global view of mutations on a chromosomal basis is provided by these plots. A higher degree of mutations are evident on chromosomes eight and 14 for both Presentation and Relapse. Chromosome 18 contains more mutations in the Relapse sample vs. Presentation. Global views may provide rapid insights, especially as newly discovered types of complex genomic rearrangements, such as chromothripsis [61], katageis and the underlying roles for APOBEC and similar family enzymes [62], have their signatures or motifs become more understood.

A scatter plot of paired samples from Presentation and Relapse are profiled in Additional File 3. The $\mathrm{x}$-axis contains the Presentation and $\mathrm{y}$-axis the Relapse, and both axes are ordered by VAF. Key genes are labelled. Color discriminates paired mutations (blue) from those appearing only in the Presentation (green) and Relapse (red). A summary illustration of the mutational landscape is provided by this graphic, namely what is common and different between the two samples. There are a noteworthy fraction of shared mutations but also a reasonable amount of variants unique to each sample.

A visual exploration of integrated mutational data revealing the evolutionary trajectories is provided by iCloneViz. Figure 1 demonstrates Gaussian kernel density plots with paired scatter plots for the Presentation (A) and Relapse (B) samples. The paired plots serve to profile the magnitude and frequency of DNA mutations found in these tumor samples. The $\mathrm{x}$-axis for each graph is VAF. The output of the kernel density function is contained on the y-axis. Peaks on the kernel density plots may infer dominant clones and subclones. A measure of relative abundance (Depth) [15] is contained on the $y$-axis of the scatter plot. Key genes are labelled, and two glyphs are used to signify if a mutation was found only in WES (blue circle) or found in both WES and RNA-seq (red star). A level of heterogeneity across the Presentation and Relapse samples is evident by the distinct peaks seen in the two kernel density plots.

iCloneViz has a number of filtering options. Figure 2 shows a series of kernel density plots with associated scatter plots for the key genes found in the Presentation (A) and Relapse (B) samples. The units associated with the $x$ and $y$ axes are unchanged. The mutational glyph assignments are also without change. Distinct peaks are evident in both samples indicating heterogeneity.

The temporal DNA-based mutational dynamics and clonal evolution observed in the Presentation and Relapse samples are shown in Figure 3A. This is an

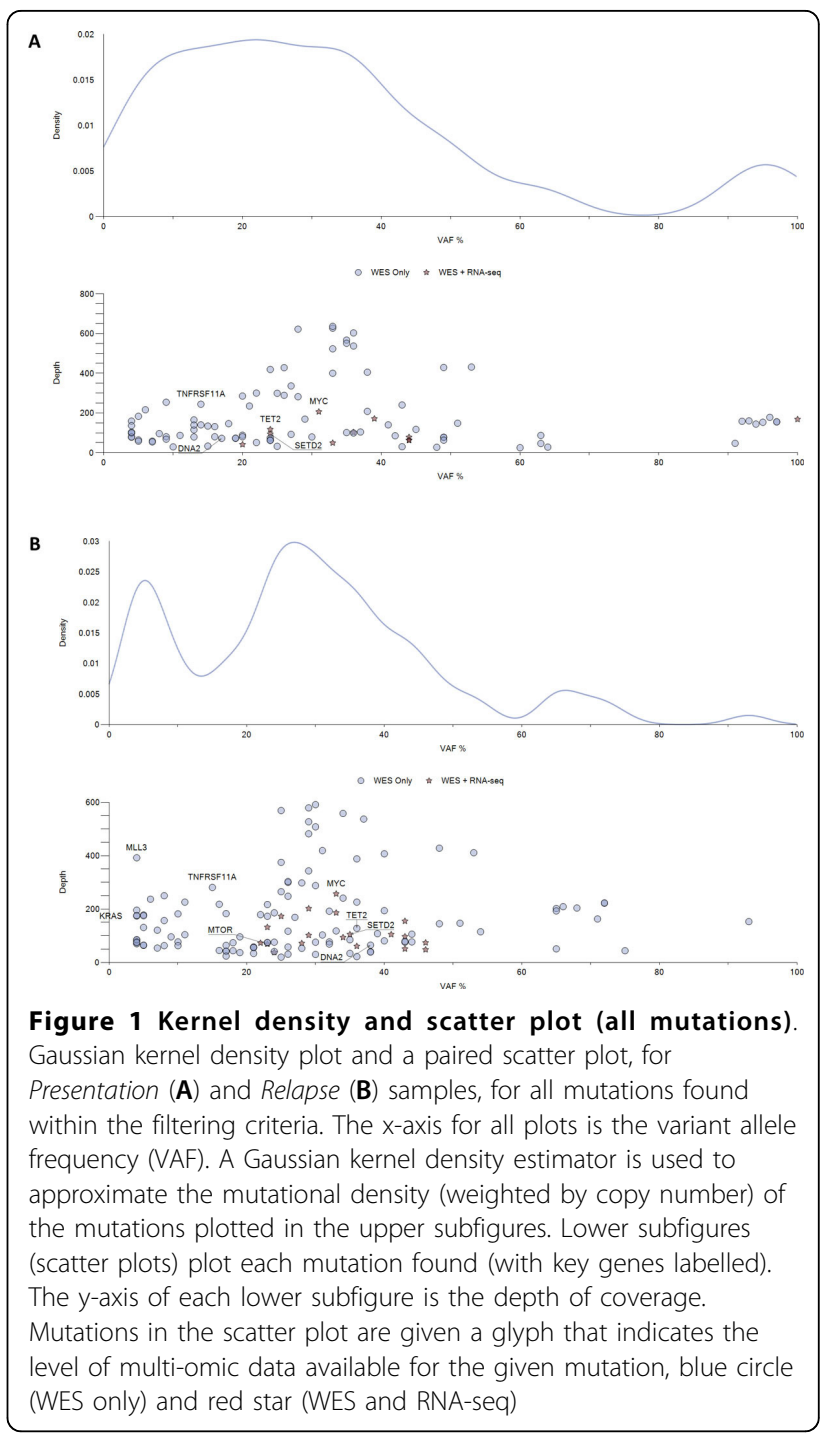

extension of the iCloneViz analysis. The Presentation sample was obtained when the patient was initially diagnosed with MM in year 2010. It contains 89 mutations that passed filtering criteria and was found to have a Shannon Diversity Index (SDI) of 3.89. There are five mutations classified from the key genes (KG) group and are: DNA2, MYC, SETD2, TET2, and TNFRS11A.

An evolutionary selection event occurred later in year 2010 , as a result of the patient receiving definitive therapy for MM. The patient did well until year 2013 when a relapse occurred and a new bone marrow aspirate was obtained. Comparing the Presentation to Relapse reveals that all the key genes survive, including the amplified $M Y C$ oncogene, which also contains a VUS missense mutation. In addition three more mutated genes are gained including the histone methyltransferase $M L L 3$, along with the KRAS and MTOR oncogenes. 


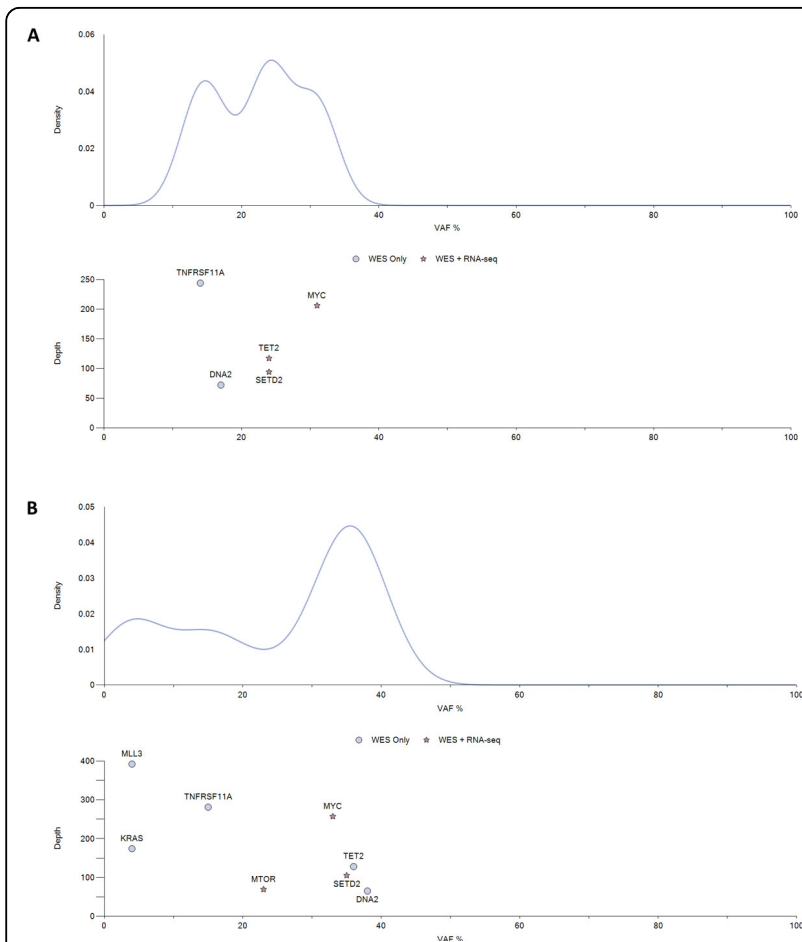

Figure 2 Kernel density and scatter plot (key genes only) Gaussian kernel density plot and a paired scatter plot for Presentation (A) and Relapse (B) samples, showing key genes found within the filtering criteria only. The $\mathrm{x}$-axis for all plots is the variant allele frequency (VAF). A Gaussian kernel density estimator is used to estimate the mutational density (weighted by copy number) of the mutations plotted in the upper subfigures. Lower subfigures (scatter plots) display each mutation found. The $y$-axis of each lower subfigure is the depth of coverage. Each mutation in the scatter plot are given a glyph that indicates the level of multi-omic data available for the given mutation. See legend for glyph assignments.

A higher number of mutations (122) are found in the Relapse sample. It also has an increased SDI (4.25), which implies more uncertainty or randomness in the process, from an information theory viewpoint [45]. From a cancer biology view, it indicates a progression of disease or diversification in the mutational landscape.

Table 1 lists the summary information for the Presentation key genes, and Table 2 for the Relapse key genes. Columns are Gene for gene symbol, DNA Mutation, AA Change for the corresponding amino acid change, $C N$ for copy number, $D P$ for depth, $A F$ for allelic frequency, RNA Mutation to indicate expressed mutations, and Notes that contains the gene class. Noted are three mutations in the Presentation group (MYC, SETD2, $T E T 2$ ), which have their DNA mutations also found to be expressed in mRNA. The Relapse key genes also contain three DNA variants found to have their mutations expressed in RNA, and include the still amplified and mutated MYC oncogene, SETD2, and the MTOR oncogene that contains a hotspot mutation (p.Val2006Leu).

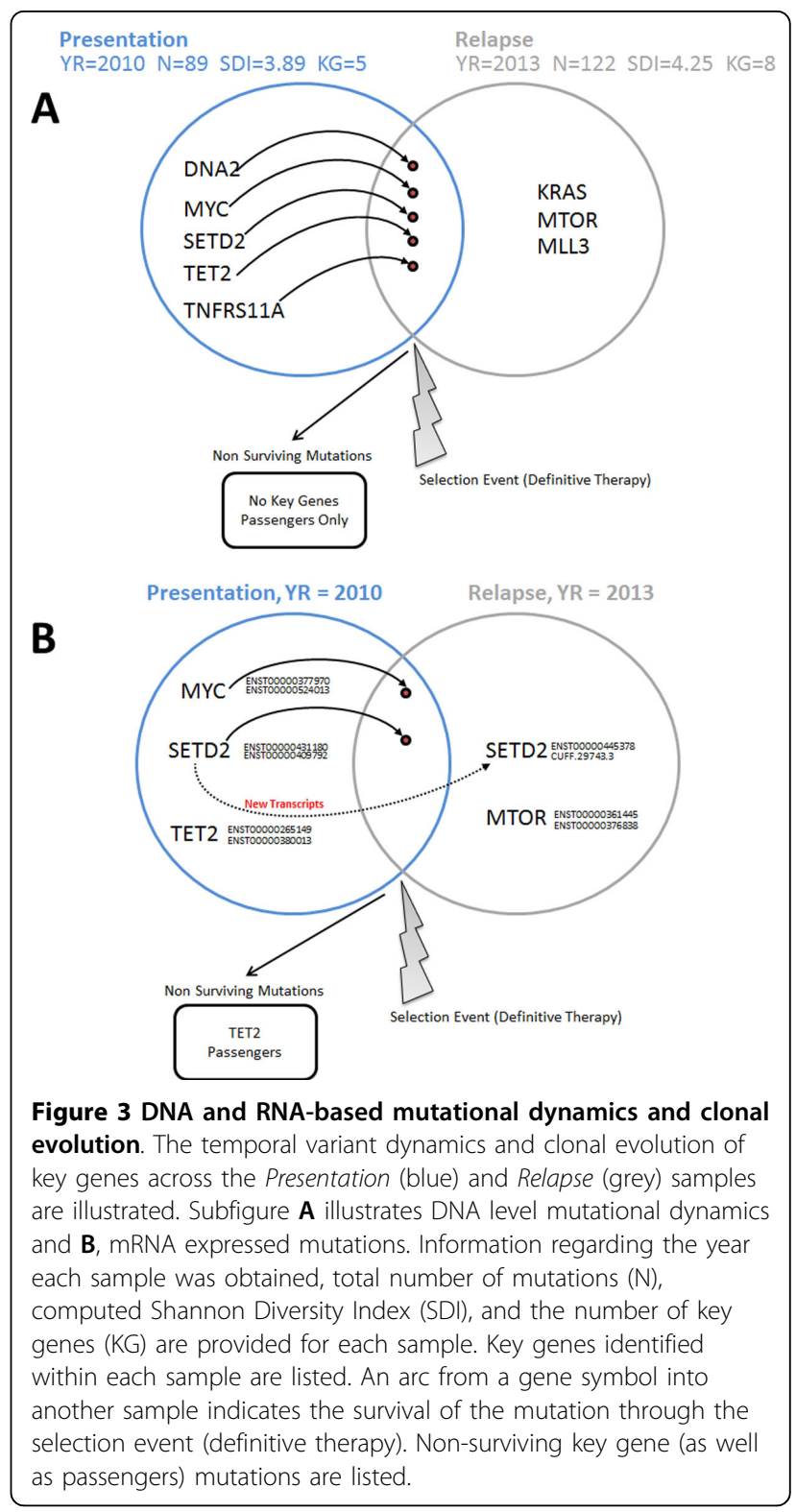

Figure 3B illustrates the temporal RNA-based mutational dynamics and clonal evolution. This is also an extension of the iCloneViz analysis. The $M Y C$ oncogene and SETD2, in addition to having their DNA mutations found in mRNA, have two transcripts in the Presentation that are also found in the Relapse sample. SETD2 also has two new transcripts appearing in the Relapse sample. CUFF.29743.3 is a novel transcript due to a new exon. However, this exon was found to have only one supporting read by IGV, and thus not seriously considered to be viable. The TET2 transcripts do not survive and are listed in the "Non Surviving Mutations" box along with passengers. MTOR, which was a new DNA mutation found in the Relapse sample, has two viable mRNA transcripts now appearing in the Relapse. Table 3 lists the expressed 
Table 1. DNA-based Presentation key genes

\begin{tabular}{llllllll}
\hline Gene & DNA Mutation & AA Change & CN & DP & AF & RNA Mutation & Notes \\
\hline DNA2 & c.146delG & & 1 & 82 & 17 & N & DNA repair \\
\hline MYC & c.226G $>$ A & p.Ala76Thr & 3 & 207 & 31 & Y & Amp oncogene + VUS \\
\hline SETD2 & c.7085A $>$ G & p.Gln2362Arg & 1 & 94 & 24 & HMT & Oncogene \\
\hline TET2 & c.2725C $>$ T & p.Gln909* & 3 & 117 & 24 & $Y$ & Bone remodelling \\
\hline TNFRSF11A & c.1097C $>$ T & p.Pro366Leu & 3 & 244 & 14 & N
\end{tabular}

A tabular version of the clonal dynamics displayed in Figure $3 \mathrm{~A}$ lists changes in allelic frequency, depth and copy number for the Presentation sample. Also indicated is whether or not the DNA mutation was expressed in the sample's associated mRNA. Abbreviations: CN (Copy Number), DP (Depth), Allelic Frequency (AF), VUS (Variant of Uncertain Significance), HMT (Histone Methyltransferase), AA (Amino Acid) and Amp (Amplified).

Table 2. DNA-based Relapse key genes

\begin{tabular}{|c|c|c|c|c|c|c|c|}
\hline Gene & DNA Mutation & AA Change & $\mathrm{CN}$ & $\mathrm{DP}$ & $\mathrm{AF}$ & RNA Mutation & Notes \\
\hline$\overline{\mathrm{DNA} 2}$ & Same & same & 3 & 70 & 38 & $\mathrm{~N}$ & \\
\hline MYC & Same & same & 3 & 257 & 33 & Y & \\
\hline SETD2 & Same & same & 2 & 105 & 35 & $Y$ & \\
\hline TET2 & Same & same & 3 & 129 & 36 & $\mathrm{~N}$ & \\
\hline TNFRSF11A & Same & same & 3 & 283 & 15 & $\mathrm{~N}$ & \\
\hline KRAS & c. $183 A>C$ & p.Gln61His & 3 & 175 & 4 & $\mathrm{~N}$ & Oncogene \\
\hline$\overline{\text { MTOR }}$ & c. $6016 G>C$ & p.Val2006Leu & 1 & 69 & 23 & $Y$ & Oncogene \\
\hline MLL3 & $c .944 G>A$ & p.Gly315Asp & 1 & 392 & 4 & $\mathrm{~N}$ & HMT \\
\hline
\end{tabular}

A tabular version of the clonal dynamics showed in Figure $3 \mathrm{~A}$ lists changes in allelic frequency, depth and copy number for the Relapse sample.

Table 3. mRNA expression of key gene mutations in the Presentation sample

\begin{tabular}{lllll}
\hline Gene & DP & AF & Transcript & FPKM \\
\hline MYC & 192 & 100 & ENST00000377970 & 42 \\
\hline MYC & 192 & 100 & ENST00000524013 & 58 \\
\hline SETD2 & 25 & 50 & ENST00000409792 & 9.5 \\
\hline SETD2 & 25 & 50 & ENST00000431180 & 2.2 \\
\hline TET2 & 6 & 50 & ENST00000380013 & 2.0 \\
\hline TET2 & 6 & 50 & ENST00000265149 & 1.2 \\
\hline
\end{tabular}

A tabular version of the clonal dynamics as shown in Figure 3B, lists changes in depth, allelic frequency and FPKM in the Presentation sample.

Abbreviations: DP (Depth) and Allelic Frequency (AF).

RNA mutated key gene transcripts for the Presentation sample and Table 4 for Relapse.

Table 5 lists the DNA methylation of tumor suppressor genes (TSGs) for the Presentation sample passing iCloneViz filtering criteria from $\sim 485,000$ possible candidates. The filtering criteria consisted of: i) Avg-Beta $\geq$ 25\%, ii) "Promoter Associated" regulatory feature, and iii) being from a CpG Island. The methylation data set was integrated with RNA-seq to directly associate the raw (unnormalized) RNA Read Counts for the TSGs identified by filtering criteria. The majority of TSGs in the Presentation (Table 5) and Relapse (Table 6) have zero or very low read counts, indicating a lack of mRNA expression and thus no protein production. Loss of TSG(s) is associated with both the onset and progression of many cancers $[63,64]$.
Table 4. mRNA expression of key gene mutations in the Relapse sample

\begin{tabular}{lllll}
\hline Gene & DP & AF & Transcript & FPKM \\
\hline MYC & 100 & 100 & ENST00000377970 & 24 \\
\hline MYC & 100 & 100 & ENST00000524013 & 55 \\
\hline SETD2 & 29 & 50 & ENST00000409792 & 3.2 \\
\hline SETD2 & 29 & 50 & ENST00000431180 & 1.5 \\
\hline SETD2 & 29 & 50 & ENST00000445387 & 1.2 \\
\hline SETD2 & 29 & 50 & CUFF.29743.3 & 9.4 \\
\hline MTOR & 20 & 50 & ENST00000361445 & 4.7 \\
\hline MTOR & 20 & 50 & ENST00000376838 & 2.2 \\
\hline
\end{tabular}

A tabular version of the clonal dynamics as shown in Figure $3 \mathrm{~B}$, lists changes in depth, allelic frequency and FPKM for the Relapse sample.

Why did the patient's cancer recur following definitive therapy? Does the experimental information from DNA, RNA, and methylation analyses provide answers or insights concerning the relapse? Regarding the Presentation sample, associating the findings from Figure 1A, and Figure 2A, the founder clone contains an amplified and mutated $M Y C$ oncogene. Table 1 reports a missense mutation in $M Y C$ having the base substitution of c.226G $>$ A, resulting in alanine being replace by threonine at position 76 (p.Ala76Thr) in the amino acid chain. The copy number is 3 (amplified), depth of coverage is 207 and the allelic fraction is 31 . This mutation is also expressed in mRNA. Additional File 4 shows a MYC lolliplot diagram made by the Protein Paint [65] application 
Table 5. DNA methylation of TSGs in the Presentation sample

\begin{tabular}{|c|c|c|c|c|c|c|c|}
\hline Target ID & Avg-Beta & Gene & Ensembl GenelD & RNA Read Count & Classification & Regulatory Feature & Relation to CpG Island \\
\hline $\operatorname{cg} 27549619$ & 0.3934072 & AXIN1 & ENSG00000103126 & 4 & TSG & Promoter Associated & Island \\
\hline $\operatorname{cg} 26370022$ & 0.6804651 & BRCA1 & ENSG00000012048 & 0 & TSG & Promoter Associated & Island \\
\hline $\operatorname{cg} 11529738$ & 0.7789396 & BRCA1 & ENSG00000012048 & 0 & TSG & Promoter Associated & Island \\
\hline cg24900425 & 0.9020266 & BRCA1 & ENSG00000012048 & 0 & TSG & Promoter Associated & Island \\
\hline $\operatorname{cg} 13601799$ & 0.2905587 & CDKN2A & ENSG00000147889 & 13 & TSG & Promoter Associated & Island \\
\hline $\operatorname{cg} 01437571$ & 0.3243266 & CEBPA & ENSG00000245848 & 0 & TSG & Promoter Associated & Island \\
\hline cg00976692 & 0.291538 & MEN1 & ENSG00000133895 & 8 & TSG & Promoter Associated & Island \\
\hline $\operatorname{cg} 14803009$ & 0.3387372 & $\mathrm{MSH} 2$ & ENSG00000095002 & 0 & TSG & Promoter Associated & Island \\
\hline $\operatorname{cg} 22866426$ & 0.4628801 & RUNX1 & ENSG00000159216 & 1 & TSG & Promoter Associated & Island \\
\hline $\operatorname{cg} 27003951$ & 0.2562254 & SOCS1 & ENSG00000185338 & 0 & TSG & Promoter Associated & Island \\
\hline
\end{tabular}

Listed are the DNA methylation of tumor suppressor genes (TSGs) passing iCloneViz filtering criteria from 485,000 possible candidates, for the Presentation sample. The filtering criteria consisted of: i) Avg-Beta $\geq 25 \%$, ii) "Promoter Associated" regulatory feature, and iii) being from a CpG Island. The methylation data set was integrated with RNA-seq to directly associate the raw (unnormalized) RNA Read Counts for the identified TSGs.

Table 6. DNA methylation of TSGs in the Relapse sample

\begin{tabular}{|c|c|c|c|c|c|c|c|}
\hline Target ID & Avg-Beta & Gene & Ensembl Gene ID & RNA Read Count & Classification & Regulatory Feature & Relation to CpG Island \\
\hline cg27549619 & 0.443731 & AXIN1 & ENSG00000103126 & 0 & TSG & Promoter Associated & Island \\
\hline cg02086790 & 0.4921295 & AXIN1 & ENSG00000103126 & 0 & TSG & Promoter Associated & Island \\
\hline cg26370022 & 0.6747859 & BRCA1 & ENSG00000012048 & 0 & TSG & Promoter Associated & Island \\
\hline cg24900425 & 0.8910863 & BRCA1 & ENSG00000012048 & 0 & TSG & Promoter Associated & Island \\
\hline cg11529738 & 0.7637555 & BRCA1 & ENSG00000012048 & 0 & TSG & Promoter Associated & Island \\
\hline $\operatorname{cg} 13601799$ & 0.467826 & CDKN2A & ENSG00000147889 & 38 & TSG & Promoter Associated & Island \\
\hline cg00976692 & 0.293402 & MEN1 & ENSG00000133895 & 0 & TSG & Promoter Associated & Island \\
\hline cg14803009 & 0.4267139 & $\mathrm{MSH} 2$ & ENSG00000095002 & 0 & TSG & Promoter Associated & Island \\
\hline cg22073802 & 0.2875548 & PTCH1 & ENSG00000185920 & 23 & TSG & Promoter Associated & Island \\
\hline cg22866426 & 0.5251715 & RUNX1 & ENSG00000159216 & 0 & TSG & Promoter Associated & Island \\
\hline cg17339910 & 0.399428 & RUNX1 & ENSG00000159216 & 0 & TSG & Promoter Associated & Island \\
\hline cg04004558 & 0.2809271 & SOCS1 & ENSG00000185338 & 0 & TSG & Promoter Associated & Island \\
\hline $\operatorname{cg} 27003951$ & 0.2598022 & SOCS1 & ENSG00000185338 & 0 & TSG & Promoter Associated & Island \\
\hline
\end{tabular}

Listed are the DNA methylation of tumor suppressor genes (TSGs) passing iCloneViz filtering criteria from 485,000 possible candidates, for the Relapse sample. Filtering criteria and integration with RNA-seq is the same as is reported for the Presentation sample (Table 5).

showing the possible missense mutations, depending on splicing, in the coding region. Table 3 shows the $M Y C$ transcripts with good depth of coverage and FPKMs. Finally, Table 5 shows four TSGs (BRCA1, CEBPA, $M S H 2$, SOCS1) with a CpG Island, promoter associated regulatory feature being methylated by more than $25 \%$ and having RNA read counts of zero, indicating these genes have been silenced.

Concerning the Relapse sample, integrating findings from Figure 1, B and Figure 2, B shows that the founder clone continues to contain the mutated $M Y C$ oncogene. Table 2 reports the same $M Y C$ DNA mutation, the copy number is still amplified at three, DP is 257 , allelic frequency is $33 \%$, and the mutation is present in mRNA. The MTOR oncogene is new, and contains a hotspot mutation (c.6016G $>$ C, p.Val2006Leu), which is also found in RNA. Figure 3B shows survival of $M Y C$ related transcripts from Presentation to Relapse, and the transcripts for MTOR. Table 4 shows the two MYC transcripts with good depth of coverage and FPKMs, and the $M T O R$ transcripts have reasonable values. Table 6 displays six TSGs (AXIN1, BRCA1, MEN1, MSH2, RUNX1, SOCS1) with CpG Island promoter associated regulatory feature being methylated by more than $25 \%$ and having RNA read counts of zero, indicating a progression of gene silencing. The KRAS oncogene appears in the Relapse but the mutation was not expressed in RNA (Table 2) therefore would not be considered for therapeutic targeting.

Deregulated expression of $M Y C$ is a hallmark feature of cancer and serves to uncouple growth factor dependent proliferation [66], and may occur through a variety of mechanisms (e.g., gene amplification, translocation, focal enhancer amplification, or constitutive activation of 
upstream signalling pathways) [67]. $M Y C$ over-expression occurs in $\sim 30 \%$ of human cancers and commonly is a harbinger for a poor clinical outcome, aggressive biological behavior, increased chance of relapse and advanced stage of disease at initial diagnosis [68]. Studies in transgenic mouse models have identified $M Y C$ inactivation leads to prompt tumor regressions [69].

There are now a number of new agents in clinical trials for targeting $M Y C$, for instance the BET bromodomain inhibition [70,71], and this should be considered for subsequent therapy. Additionally, the Relapse sample contained a hotspot mutation in MTOR and targeting with a "rapalog" (rapamycin and its analogs) should also be considered. Precision medicine and therapeutic combinations with new and more targeted agents are challenging and an active area in clinical trials and translational research $[72,73]$. Essential to these efforts are advanced bioinformatics with abilities to integrate multi-omic datasets, combat cancer heterogeneity via clonal and evolutionary approaches, and ultimately provide clinical utility thru an improved understanding of the disease process at hand. This was demonstrated in this study.

\section{Conclusions}

Illustrated in this study has been the temporal-based analysis of integrated clonal dynamics of a single patient with MM by examining the Presentation and Relapse tumor samples. This involved the visualization and quantification of variant/mutational dynamics in the context of integrated evolution of WES, RNA-seq, and DNA methylation. Subpopulations of mutations will evolve over time due to natural selection events related to cell intrinsic or micro-environmental factors, as well as selection events induced therapeutically. Selection events eliminate some mutations and provide a survival advantage to others. iCloneViz provides global views of mutational events as well as rapid data integration of $\mathbf{i}$ ) WES, ii) RNA-seq, and iii) DNA methylation. This results in an enriched picture with a more focused specificity of mutational events, and provides evidence and more confidence for therapeutic assignments.

Heterogeneity is found in many cancers and limits aggregate approaches for scientific and clinical utility. MM is known as a heterogeneous cancer with a complex molecular landscape. Clonality and integrated genomics has been advocated as means to combat heterogeneity $[59,60]$. The illustrated novelty and precise contribution of $\mathrm{iCloneViz}$ is the ability to perform integrative clonality analysis utilizing data sets from: i) WES, ii) RNA-seq, and iii) DNA methylation. To date, there is no other software based clonality tool available (commercially or open source) that can perform integrative clonality analysis. Given the repeated findings of multiple TCGA studies reporting heterogeneity with a complex mutational landscape $[14,74]$, additional analytical approaches are needed; since it is evident that DNA analysis is necessary but not sufficient. Our approach, which builds on previous work now employs clonality and integrative genomics, both of which have been recommended to combat heterogeneity.

In this study, observed in all serial MM samples was the presence of an amplified $M Y C$ oncogene species with a VUS missense mutation. $M Y C$ is a transcription factor and master regulator of $\sim 15 \%$ of all gene expression, and also functions to regulate chromatin structure. A permanent remission or cure was not achieved despite definitive therapy. The dominant genetic alterations in the founder clone was never targeted specifically for therapy thus cure or a lasting remission was unlikely. For major advances in cancer management, a systematic approach to collect tissue samples at diagnosis, and serially at relapse(s), in order to profile the dynamic clonal evolution is critical.

\section{Appendix \\ iCloneViz pseudocode}

The pseudocode for iCloneViz is now presented, and is described and documented using a PDL (Program Design Language) structure as described in Pressman [75]. A C-style notation was utilized. A flow diagram of the pseudocode is shown in Additional File 5. The multi-omic relational integration is illustrated in Additional File 1.

Symbol Definitions:

- patient_id: Patient identifier.

- button_selection: An instance of the button that was clicked.

- button_click_filter: Boolean indicating whether an instance of the button labeled "Filter" was clicked.

- button_click_show_tsg_methylation_table: Boolean indicating whether an instance of the button labeled "Show TSG Methylation Table" was clicked.

- button_click_show_wes_table: Boolean indicating whether an instance of the button labeled "Show WES Table" was clicked.

- button_click_show_rna_table: Boolean indicating whether an instance of the button labeled "Show RNA Table" was clicked.

- exp_id []: Array of one or two patient experiment identifiers. Can be referenced via subscripting (e.g., exp_id [0]).

- exp_id $[i] . W$ : WES data in relation $W$ for the patient experiment with the experiment identifier exp_id [i]. 
- filter_settings: Data structure containing all filter settings including:

$\circ$ min vaf: Minimum variant allele frequency (default 4\%).

$\circ$ max_vaf : Maximum variant allele frequency (default 100\%).

$\circ$ min_depth: Minimum read depth (default 20).

$\circ$ max_depth: Maximum read depth (default 1000).

0 min meth: Minimum methylation percent (default 25\%).

opacity: Opacity of scatter plot points (default 50\%).

$\circ$ show_kg_only: Boolean indicating whether to show only mutations found in the key genes list $K$ (default False).

- default_filter_settings: Default values used for filtering.

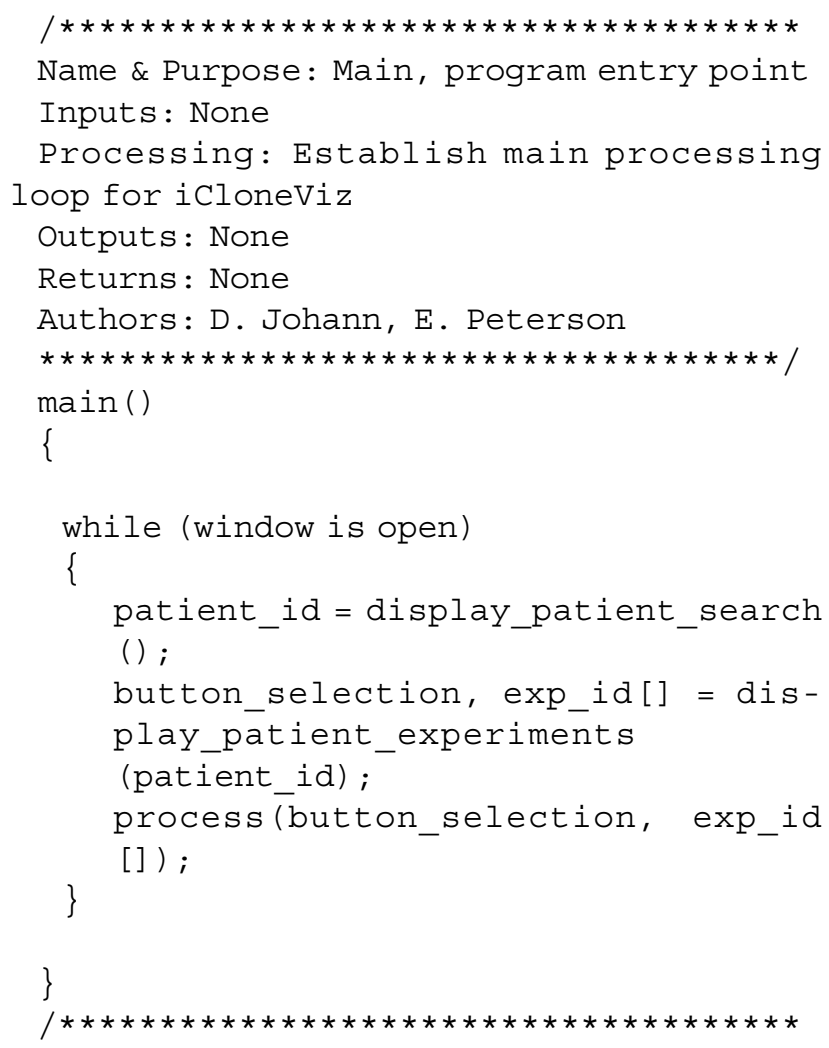

Name \& Purpose: Display Patient Search, via patient ID display patient meta-data

Inputs: none

Processing: Display patient meta-data

Outputs: None

Returns: ID of selected patient from MPMDB

Authors: D. Johann, E. Peterson

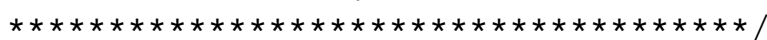

display_patient_search()

\{

patient_id = input from user;

- get / display patient meta-data via relation $P$;

return patient_id;

\}

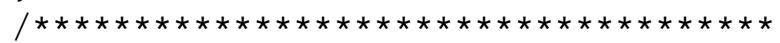

Name \& Purpose: Display Patient Experiments, show available experiments for iCloneViz analysis

Inputs: Patient ID

Processing: Retrieve patient experimental meta-data from MPMDB

Outputs: Patient experimental data now in memory

Returns: Array of experiment IDs, Button selection for selected analysis, eg, Genomic Real Estate or Paired Scatter Plot or KDE plus Scatter Plot

Authors: D. Johann, E. Peterson

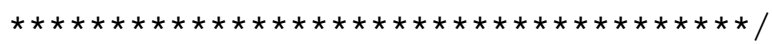

display_patient_experiments

(patient_id)

\{

- get / display all experimental data from database (MPMDB) for patient via relation $P$;

exp_id[] = selected experiment ids;

return button_selection, exp_id[]

\}

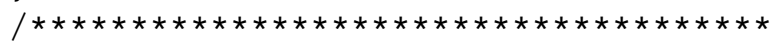

Name \& Purpose: Process, process data and display visualization based on the user's choice of visualization

Inputs: Button selection, \& Experiment IDs

Processing: Execute specific function to handle processing based on user's choice of visualization

Outputs: None

Returns: None

Authors: D. Johann, E. Peterson

$* * * * * * * * * * * * * * * * * * * * * * * * * * * * * * * * * * * * * 1$

process (button_selection, exp_id [])

\{

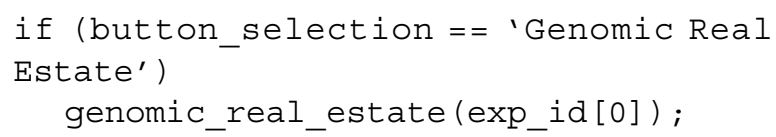




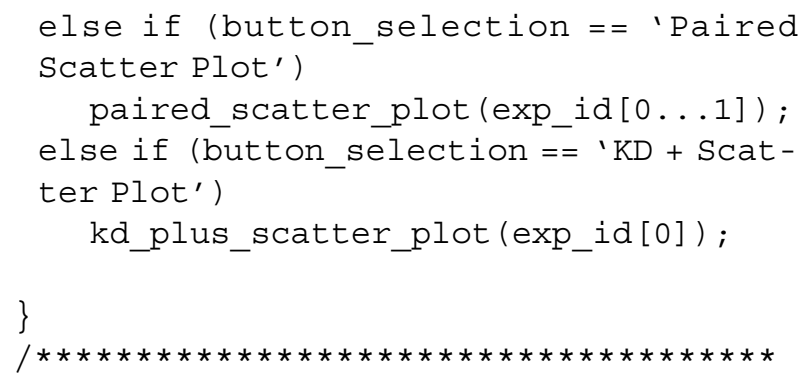

Inputs: Experiment ID of experiment to visualize

Processing: Using R.NET API, generate plot image and display in window

Outputs: Scatter plot of all mutations by chromosome and variant allele frequency, read depth is encoded by color, see Additional File 2

Returns: None

Authors: D. Johann, E. Peterson

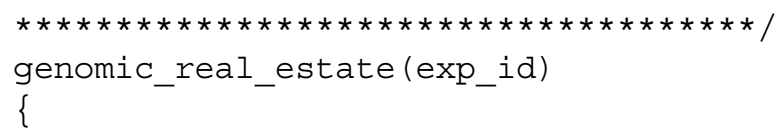

Name \& Purpose: Paired Scatter Plot, fetch filter settings and call function to display paired scatter plot

Inputs: Experiment IDs

Processing: If first time displaying, use default filter settings to display paired plot, otherwise, fetch filter settings from user and display paired plot

Outputs: None

Returns: None

Authors: D. Johann, E. Peterson

$\star * * * * * * * * * * * * * * * * * * * * * * * * * * * * * * * * * * * * /$

paired_scatter_plot (exp_id [])

\{

display_paired_scatter_plot (default_filter settings, exp_id[]): while (window is open)

\{

filter settings = read filter toolbar () ; display paired scatter plot(filter_settings, exp_id[]);

\}

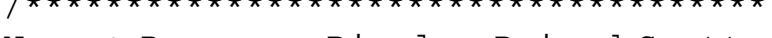

Name \& Purpose: Display Paired Scatter Plot, calculate WES mutations in common and exclusive to each experiment and generate paired scatter plot

Inputs: Filter settings fetched from user input, and Experiment IDs

Processing: Fetch WES mutations, and using filter settings and referenced equations, calculate mutations in common and exclusive to each experiment; display paired scatter plot with data, see Additional File 3

Outputs: A paired scatter plot for each experiment in exp_id array, based on variant allele frequency

Returns: None

Authors: D. Johann, E. Peterson

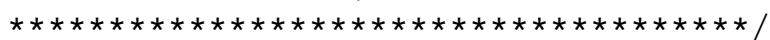
display_paired_scatter_plot(filter_settings, exp id [])

\{

// Relational algebra Eq. (9) common $=\exp$ id [0].W $\mathrm{W} \exp$ id [1].W ;

// Relational algebra Eq. (10) exp0_unique $=\exp \_i d[0] \cdot W \backslash$ exp_id [1] . $\mathrm{W}$;

// Relational algebra Eq. (10) exp1_unique $=$ exp_id [1].W $\backslash$ exp_id[0] . $\mathrm{W}$;

- label all genes in $K$;

if (filter_settings.show_kg_only = = true) 
- hide all non-labelled points;

- plot common, exp0_unique, and exp1_unique based on DNAllelicFreq (variant allelic frequency) using filtersettings ;

\}

/

Name \& Purpose: Read Filter Toolbar, gather user-defined filter settings, and display "TSG Methylation Table", "WES Table", or "RNA Table" if the user so desires

Inputs: None

Processing: Collect filter settings from user; if the user clicks on "Filter" the filter settings are returned and they are used when displaying a desired plot; if the user clicks on "Show TSG Methylation Table", "Show WES Table", or "Show RNA Table", the desired table is displayed using the referenced equations

Outputs: Tables selected if clicked

Returns: User-defined filter settings

Authors: D. Johann, E. Peterson

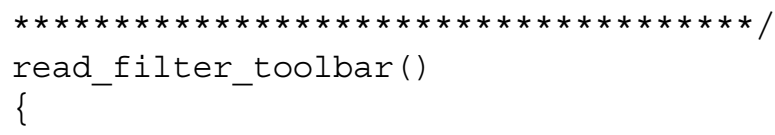

while (true)

\{

filter_settings.min_vaf = user input minimum VAF;

filter_settings.max_vaf = user input maximum VAF;

filter_settings.min_depth = user input minimum read depth;

filter_settings.max_depth = user input maximum read depth;

filter_settings.min_meth = user input minimum methylation percent;

filter_settings.opacity = user input scatter plot point opacity;

filter_settings.show_kg_only = user

input show key gene only;

if (button_click_filter)

return filter_settings;

else if (button_click_show_tsg_ methylation_table)

- display TGS Methylation Table via

Relational algebra Eq. (13);

break;

else if (button_click_show_wes_table)

- display WES Table Eq. (11);

\author{
break; \\ else if (button_click_show_rna_table) \\ - display RNA Table Eq. (14); \\ break; \\ \} \\ return filter_settings;
}

\}

Name \& Purpose: KD Plus Scatter Plot, call subroutines to process and render data for each of the individual plot areas, as well as, to calculate various metircs

Inputs: Experiment ID of experiment to visualize

Processing: Call subroutines to process and render the individual plot areas; call subroutine to calculate various metrics

Outputs: None

Returns: None

Authors: D. Johann, E. Peterson

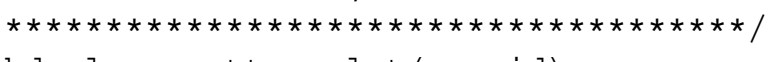

kd_plus_scatter_plot (exp_id)

\{

display_kd_plot(default_filter_settings, exp_id);

display_scatter_plot(default_filter_settings, exp_id);

calculate_metrics(default_filter_settings, exp_id);

while (window is open)

\{

filter_settings = read_filter_toolbar ();

display_kd_plot(filter_settings, exp_id);

display_scatter_plot(filter_settings, exp_id);

calculate_metrics(filter_settings, exp_id);

\}

\}

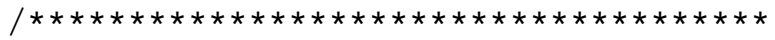

Name \& Purpose: Display KD Plot, displays the kernel density estimation curve, using the ' $k s^{\prime}$ R package to calculate an appropriate bandwidth

Inputs: User-defined filter settings and the experiment ID to be visualized

Processing: Calculate the KDE for DNA mutations, use R.NET to utilize the 'ks' package (used for bandwidth calculation) 
Outputs: KD curve, see Figures $1 \& 2$

Returns: None

Authors: D. Johann, E. Peterson

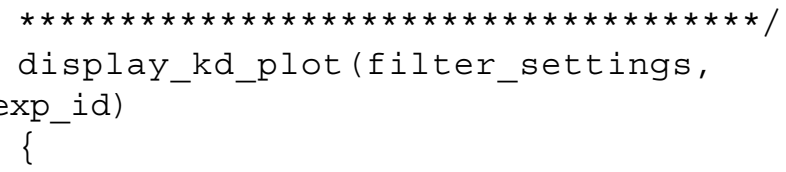

- calculate KDE based on Relational algebra Eq. (11) and Eq. (2) for all mutations in $\hat{W}$ and weighted by copy number;

- utilize R.NET to call 'hpi' function in the $\mathrm{R}$ ' $\mathrm{ks}^{\prime}$, for bandwidth calculation;

- display KD plot using filter settings;

\}

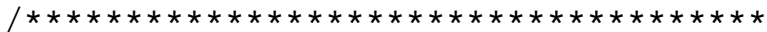

Name \& Purpose: Display Scatter Plot, displays the DNA mutational scatter plot, and tooltip containing RNA expression based info if available

Inputs: User-defined filter settings and the experiment ID to be visualized

Processing: Displays a scatter plot point for each DNA mutation, set each glyph depending on the degree of modality data available, and populate tooltip with RNA expression data if available

Outputs: Mutational scatter plot, see Figures $1 \& 2$

Returns: None

Authors: D. Johann, E. Peterson

display scatter plot (filter settings, exp_id)

\{

- calculate $\otimes$ by Relational algebra Eq. (11) using filter settings;

- set glyph for all DNA mutations to be a blue circle and place along a-axis according to variant allele frequency and along y-axis by depth

for each tuple $\hat{w}$ in W having RNA data calculate $\hat{R}(\hat{w})$ by Relational algebra Eq. (12) \{

- update point glyph, (red star, expressed RNA mutation);

- build hover-over tooltip to contain: gene name, transcript(s) ids and $\operatorname{FPKM}(\mathrm{s})$ from $\hat{R}(\hat{w})$;
- display scatter plot for each mutation in $\otimes$ with associated tooltip (if RNA data is available);

\}

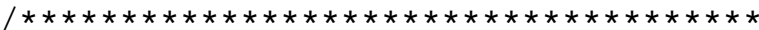

Name \& Purpose: Calculate Metrics, calculate various metrics for display

Inputs: User-defined filter settings and the experiment ID to be visualized

Processing: Calculate SDI, total number of mutations, and total number of key gene mutations, for the data being visualized

Outputs: Calculated metrics

Returns: None

Authors: D. Johann, E. Peterson

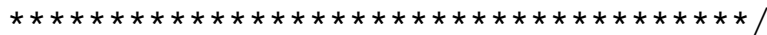

calculate_metrics(filter_settings, exp_id)

\{

- calculate SDI using Eq. (1) and relation $\otimes$ (Relational algebra Eq. (11)) using filter_settings;

- calculate total number of mutations in relation $\nabla$ using filter settings;

- calculate total number of key genes from $K$ which are found in relation $\otimes$ using filter_settings;

- display metrics;

\section{Additional material}

Additional File 1: Muti-omic relational integration. Illustration of relations used to integrate multi-omic datasets. The upper-right box (Relations \& Attributes) defines all relations and their attributes. The leftmost section (iCloneViz DB) lists each dataset and a name / identifier for each. Middle section (Multi-Omic Integration) illustrates the relationships and integration of each dataset using intermediate relations. Each intermediate integration is annotated with the attributes used in each combination. Each intermediate relation is further annotated with the equation used in the manuscript to form the given relation. The final multi-omic "Integrated Relation" is shown in the lower right.

Additional File 2: Genomic mutational overview. A genomic mutational overview of two experiments is computed and displayed. A corresponds to the Presentation sample and $\mathbf{B}$ to Relapse. These provide a general view of the inherent mutational events on a chromosomal basis. The $x$ axis contains an ordered list of chromosomes (1-22, X, Y), each sized by the number of base pairs (bp) it contains. The $y$-axis is ordered by variant allele frequency (VAF), and the color scale indicates sequence depth.

Each variant is a point in the plot.

Additional File 3: Scatter plot of paired samples. Displayed are variants in the Presentation on the $\mathrm{x}$-axis compared to Relapse on $\mathrm{y}$-axis. Both the $x$ and $y$-axes are based on VAF. Variants are colored to indicate whether they are shared or unique. See legend for color assignments. 
Additional File 4: MYC oncogene with mutation showing possible splicing events. Illustrated is a lolliplot diagram of MYC showing the possible missense mutations in the coding region depending on splicing.

Additional File 5: iCloneViz pseudocode flow diagram. Illustrated is the execution flow of iCloneViz and its associated subroutines. Each subroutine and its formal parameters is represented as a node. Each arc represents a subroutine call from one subroutine to another.

\section{Competing interests}

The authors declare that they have no competing interests.

\section{Authors' contributions}

DJJ and EAP conceived and designed the study. EAP, MAB, SSC, CJH, NW and DJJ performed experiments and analyses. DJJ and EAP designed the software. EAP, DJJ and MAB implemented the software. DJJ and EAP wrote the manuscript. All authors approved the manuscript.

\section{Declarations}

Publication of this work was supported by Program Project Grant CA 55813, from the National Institutes of Health (NIH), National Cancer Institute (NCI), Bethesda, MD, USA.

This article has been published as part of BMC Bioinformatics Volume 16 Supplement 13, 2015: Proceedings of the 12th Annual MCBIOS Conference. The full contents of the supplement are available online at http://www. biomedcentral.com/bmcbioinformatics/supplements/16/S13.

\section{Published: 25 September 2015}

\section{References}

1. Greaves M, Maley CC: Clonal evolution in cancer. Nature 2012, 481(7381):306-313

2. Vogelstein B, Papadopoulos N, Velculescu VE, Zhou S, Diaz LA, Kinzler KW: Cancer genome landscapes. science 2013, 339(6127):1546-1558.

3. Potter NE, Ermini L, Papaemmanuil E, Cazzaniga G, Vijayaraghavan G, Titley I, Ford A, Campbell P, Kearney L, Greaves M: Single-cell mutational profiling and clonal phylogeny in cancer. Genome research 2013, 23(12):2115-2125.

4. Greaves M, Maley CC: Clonal evolution in cancer. Nature 2012, 481(7381):306-313.

5. Morgan GJ, Walker BA, Davies FE: The genetic architecture of multiple myeloma. Nat Rev Cancer 2012, 12(5):335-348.

6. Greaves M: Darwinian medicine: a case for cancer. Nature Reviews Cancer 2007, 7(3):213-221.

7. Kyle RA, Rajkumar SV: Multiple myeloma. N Engl J Med 2004, 351(18):1860-1873

8. Barlogie B, Jagannath S, Desikan KR, Mattox S, Vesole D, Siegel D, Tricot G, Munshi N, Fassas A, Singhal S, et al: Total therapy with tandem transplants for newly diagnosed multiple myeloma. Blood 1999, 93(1):55-65.

9. Rajkumar SV: Treatment of multiple myeloma. Nat Rev Clin Oncol 2011, 8(8):479-491.

10. Richardson PG, Sonneveld P, Schuster MW, Irwin D, Stadtmauer EA, Facon T, Harousseau JL, Ben-Yehuda D, Lonial S, Goldschmidt H, et al: Bortezomib or high-dose dexamethasone for relapsed multiple myeloma. $N$ Engl J Med 2005, 352(24):2487-2498

11. Singhal S, Mehta J, Desikan R, Ayers D, Roberson P, Eddlemon P, Munshi N, Anaissie E, Wilson C, Dhodapkar M, et al: Antitumor activity of thalidomide in refractory multiple myeloma. N Engl J Med 1999, 341(21):1565-1571.

12. Kyle RA, Rajkumar SV: An overview of the progress in the treatment of multiple myeloma. Expert Rev Hematol 2014, 7(1):5-7.

13. Chapman MA, Lawrence MS, Keats JJ, Cibulskis K, Sougnez C, Schinzel AC, Harview CL, Brunet JP, Ahmann GJ, Adli M, et al: Initial genome sequencing and analysis of multiple myeloma. Nature 2011, 471(7339):467-472

14. Lohr JG, Stojanov P, Carter SL, Cruz-Gordillo P, Lawrence MS, Auclair D, Sougnez C, Knoechel B, Gould J, Saksena G, et al: Widespread genetic heterogeneity in multiple myeloma: implications for targeted therapy. Cancer Cell 2014, 25(1):91-101.
15. Miller CA, White BS, Dees ND, Griffith M, Welch JS, Griffith OL, Vij R, Tomasson MH, Graubert TA, Walter MJ, et al: SciClone: inferring clonal architecture and tracking the spatial and temporal patterns of tumor evolution. PLoS Comput Biol 2014, 10(8):e1003665.

16. Roth A, Khattra J, Yap D, Wan A, Laks E, Biele J, Ha G, Aparicio S, BouchardCote A, Shah SP: PyClone: statistical inference of clonal population structure in cancer. Nat Methods 2014, 11(4):396-398.

17. Strino F, Parisi F, Micsinai M, Kluger $Y$ : TrAp: a tree approach for fingerprinting subclonal tumor composition. Nucleic Acids Res 2013, 41(17):e165.

18. Zare H, Wang J, Hu A, Weber K, Smith J, Nickerson D, Song C, Witten D, Blau CA, Noble WS: Inferring clonal composition from multiple sections of a breast cancer. PLoS Comput Biol 2014, 10(7):e1003703.

19. Hajirasouliha I, Mahmoody A, Raphael BJ: A combinatorial approach for analyzing intra-tumor heterogeneity from high-throughput sequencing data. Bioinformatics 2014, 30(12):i78-86.

20. Oesper L, Mahmoody A, Raphael BJ: THetA: inferring intra-tumor heterogeneity from high-throughput DNA sequencing data. Genome Biol 2013, 14(7):R80.

21. Deshwar AG, Vembu S, Yung CK, Jang GH, Stein L, Morris Q: PhyloWGS: reconstructing subclonal composition and evolution from wholegenome sequencing of tumors. Genome Biol 2015, 16:35.

22. Peterson EA, Chavan SS, Bauer MA, Heuck CJ, Johann DJ: Revealing the inherent heterogeneity of human malignancies by variant consensus strategies coupled with cancer clonal analysis. BMC bioinformatics 2014, 15(Suppl 11):S9.

23. Zhan F, Hardin J, Kordsmeier B, Bumm K, Zheng M, Tian E, Sanderson R, Yang $Y$, Wilson C, Zangari M, et al: Global gene expression profiling of multiple myeloma, monoclonal gammopathy of undetermined significance, and normal bone marrow plasma cells. Blood 2002, 99(5):1745-1757.

24. CASAVA Support. [http://support.illumina.com/sequencing/ sequencing_software/casava.html].

25. FastQC: A Quality Control tool for High Throughput Sequence Data. [http://www.bioinformatics.babraham.ac.uk/projects/fastac/].

26. Li H, Durbin R: Fast and accurate short read alignment with BurrowsWheeler transform. Bioinformatics 2009, 25(14):1754-1760.

27. Lunter G, Goodson M: Stampy: a statistical algorithm for sensitive and fast mapping of Illumina sequence reads. Genome Res 2011, 21(6):936-939.

28. Garcia-Alcalde F, Okonechnikov K, Carbonell J, Cruz LM, Gotz S, Tarazona S, Dopazo J, Meyer TF, Conesa A: Qualimap: evaluating next-generation sequencing alignment data. Bioinformatics 2012, 28(20):2678-2679.

29. Picard Tools. [http://broadinstitute.github.io/picard/].

30. DePristo MA, Banks E, Poplin R, Garimella KV, Maguire JR, Hartl C, Philippakis AA, del Angel G, Rivas MA, Hanna M, et al: A framework for variation discovery and genotyping using next-generation DNA sequencing data. Nat Genet 2011, 43(5):491-498.

31. Sathirapongsasuti JF, Lee H, Horst BA, Brunner G, Cochran AJ, Binder S, Quackenbush J, Nelson SF: Exome sequencing-based copy-number variation and loss of heterozygosity detection: ExomeCNV. Bioinformatics 2011, 27(19):2648-2654.

32. Saunders CT, Wong WS, Swamy S, Becq J, Murray LJ, Cheetham RK: Strelka: accurate somatic small-variant calling from sequenced tumor-normal sample pairs. Bioinformatics 2012, 28(14):1811-1817.

33. Cingolani P, Platts A, Wang le L, Coon M, Nguyen T, Wang L, Land SJ, Lu X, Ruden DM: A program for annotating and predicting the effects of single nucleotide polymorphisms, SnpEff: SNPs in the genome of Drosophila melanogaster strain w1118; iso-2; iso-3. Fly (Austin) 2012, 6(2):80-92.

34. Bolger $A M$, Lohse $M$, Usadel B: Trimmomatic: a flexible trimmer for Illumina sequence data. Bioinformatics 2014.

35. Kim D, Pertea G, Trapnell C, Pimentel H, Kelley R, Salzberg SL: TopHat2: accurate alignment of transcriptomes in the presence of insertions, deletions and gene fusions. Genome Biol 2013, 14(4):R36.

36. Li H, Handsaker B, Wysoker A, Fennell T, Ruan J, Homer N, Marth G, Abecasis G, Durbin R, Genome Project Data Processing S: The Sequence Alignment/Map format and SAMtools. Bioinformatics 2009, 25(16):2078-2079.

37. Trapnell C, Williams BA, Pertea G, Mortazavi A, Kwan G, van Baren MJ, Salzberg SL, Wold BJ, Pachter L: Transcript assembly and quantification by 
RNA-Seq reveals unannotated transcripts and isoform switching during cell differentiation. Nat Biotechnol 2010, 28(5):511-515.

38. Anders S, Pyl PT, Huber W: HTSeq-a Python framework to work with high-throughput sequencing data. Bioinformatics 2015, 31(2):166-169.

39. Calling Variants in RNAseq: Methods and Workflows. [https://www. broadinstitute.org/gatk/guide/article?id=3891].

40. Dobin A, Davis CA, Schlesinger F, Drenkow J, Zaleski C, Jha S, Batut P, Chaisson M, Gingeras TR: STAR: ultrafast universal RNA-seq aligner. Bioinformatics 2013, 29(1):15-21.

41. Thorvaldsdottir H, Robinson JT, Mesirov JP: Integrative Genomics Viewer (IGV): high-performance genomics data visualization and exploration. Brief Bioinform 2013, 14(2):178-192.

42. Vogelstein B, Papadopoulos N, Velculescu VE, Zhou S, Diaz LA, Kinzler KW: Cancer genome landscapes. Science 2013, 339(6127):1546-1558.

43. [http://sciencepark.mdanderson.org/labs/wood/dna_repair_genes. html\#Human].

44. Magurran A: Measuring biological diversity Malden: Blackwell; 2004.

45. Shannon C: A mathematical theory of communication. The Bell System Technical Journal 1948, 27:379-423.

46. Almendro V, Kim HJ, Cheng YK, Gonen M, Itzkovitz S, Argani P, van Oudenaarden A, Sukumar S, Michor F, Polyak K: Genetic and phenotypic diversity in breast tumor metastases. Cancer Res 2014, 74(5):1338-1348.

47. Maley CC, Galipeau PC, Finley JC, Wongsurawat VJ, Li X, Sanchez CA, Paulson TG, Blount PL, Risques RA, Rabinovitch PS, et al: Genetic clonal diversity predicts progression to esophageal adenocarcinoma. Nat Genet 2006, 38(4):468-473.

48. Microsoft .NET Framework. [http://www.microsoft.com/net].

49. Microsoft Visual Studio. [http://www.visualstudio.com].

50. Microsoft Entity Framework. [http://msdn.microsoft.com/en-US/data/ef].

51. Microsoft SQL Server. [http://www.microsoft.com/en-US/server-cloud/ products/sql-server/default.aspx].

52. Math.NET Numerics. [http://numerics.mathdotnet.com]

53. R: A language and environment for statistical computing. [http://www.rproject.org/].

54. Tarn D: ks: Kernel density estimation and kernel discriminant analysis for multivariate data in R. Journal of Statistical Software 2007, 21(7):1-16.

55. Wand MP, Jones MC: Multivariate plugin bandwidth selection. Computational Statistics 1994, 9:97-116.

56. R.NET. [http://rdotnet.codeplex.com].

57. Garcia-Molina H, Ullman JD, Widom J: Database Systems: The Complete Book Prentice Hall Press; 2008.

58. Walker BA, Wardell CP, Melchor L, Hulkki S, Potter NE, Johnson DC, Fenwick K, Kozarewa I, Gonzalez D, Lord CJ, et al: Intraclonal heterogeneity and distinct molecular mechanisms characterize the development of $t$ $(4 ; 14)$ and $t(11 ; 14)$ myeloma. Blood 2012, 120(5):1077-1086.

59. Burgess DJ: Initially complex, always heterogeneous. NATURE REVIEWS| CanCer 2011, 11.

60. Sottoriva A, Spiteri I, Piccirillo SG, Touloumis A, Collins VP, Marioni JC, Curtis C, Watts C, Tavaré S: Intratumor heterogeneity in human glioblastoma reflects cancer evolutionary dynamics. Proceedings of the National Academy of Sciences 2013, 110(10):4009-4014.

61. Korbel JO, Campbell PJ: Criteria for inference of chromothripsis in cancer genomes. Cell 2013, 152(6):1226-1236.

62. Alexandrov LB, Nik-Zainal S, Wedge DC, Aparicio SA, Behjati S, Biankin AV, Bignell GR, Bolli N, Borg A, Børresen-Dale A-L: Signatures of mutational processes in human cancer. Nature 2013.

63. Weinberg RA: Tumor suppressor genes. Science 1991, 254(5035):1138-1146.

64. Park BH, Vogelstein B: Tumor-suppressor genes. Cancer medicine 2003, 6:87-102.

65. Protein Paint. [http://explore.pediatriccancergenomeproject.org/].

66. Gabay M, Li Y, Felsher DW: MYC activation is a hallmark of cancer initiation and maintenance. Cold Spring Harbor perspectives in medicine 2014, 4(6):a014241

67. McKeown MR, Bradner JE: Therapeutic Strategies to Inhibit MYC. Cold Spring Harbor perspectives in medicine 2014, 4(10):a014266.

68. Nesbit CE, Tersak JM, Prochownik EV: MYC oncogenes and human neoplastic disease. Oncogene 1999, 18(19):3004-3016.

69. Soucek L, Whitfield J, Martins CP, Finch AJ, Murphy DJ, Sodir NM, Karnezis AN, Swigart LB, Nasi S, Evan Gl: Modelling Myc inhibition as a cancer therapy. Nature 2008, 455(7213):679-683.
70. Mertz JA, Conery AR, Bryant BM, Sandy P, Balasubramanian S, Mele DA, Bergeron L, Sims RJ: Targeting MYC dependence in cancer by inhibiting BET bromodomains. Proceedings of the National Academy of Sciences 2011, 108(40):16669-16674.

71. Ott CJ, Kopp N, Bird L, Paranal RM, Qi J, Bowman T, Rodig SJ, Kung AL, Bradner JE, Weinstock DM: BET bromodomain inhibition targets both cMyc and IL7R in high-risk acute lymphoblastic leukemia. Blood 2012, 120(14):2843-2852.

72. Andre F, Mardis E, Salm M, Soria J-C, Siu LL, Swanton C: Prioritizing targets for precision cancer medicine. Annals of Oncology 2014, 25(12):2295-2303.

73. Estey E, Levine RL, Löwenberg B: Current challenges in clinical development of "targeted therapies": the case of acute myeloid leukemia. Blood 2015, 125(16):2461-2466.

74. Network CGA: Comprehensive molecular portraits of human breast tumours. Nature 2012, 490(7418):61-70.

75. Pressman RS: Software engineering : a practitioner's approach. 6 edition. Boston, Mass.: McGraw-Hill; 2005.

doi:10.1186/1471-2105-16-S13-S7

Cite this article as: Peterson et al:: Enhancing cancer clonality analysis with integrative genomics. BMC Bioinformatics 2015 16(Suppl 13):S7.

\section{Submit your next manuscript to BioMed Central and take full advantage of:}

- Convenient online submission

- Thorough peer review

- No space constraints or color figure charges

- Immediate publication on acceptance

- Inclusion in PubMed, CAS, Scopus and Google Scholar

- Research which is freely available for redistribution

Submit your manuscript at www.biomedcentral.com/submit
Ciomed Central 